\title{
Are Canadian plastic surgeons "shooting themselves in the foot?" A point of view
}

\author{
Arnis Freiberg MD FRCSC FACS
}

$\mathrm{I}^{\mathrm{s}}$

do realize that I belong to a different generation, and by no means do I disagree with the current generation's philosophy about life styles and the importance of paying more attention to issues outside of medicine.

However, I am seriously concerned about the future of our specialty and the future of the next generation of plastic surgeons. Who is to blame? The community or the academic surgeons? Unfortunately...both!

Not too long ago, when I was still taking call, an incident occurred that illustrates one of my concerns.

On a 'May two-four' long weekend, I received a call via Ontario 'CritiCall' from an emergency physician in a midsized town approximately $4 \mathrm{~h}$ north of Toronto. Apparently he had a young man who had sustained a partial amputation of his index finger and the emergency physician thought that the patient needed a small skin graft. Although I tried to take him through the procedure by phone, the physician did not feel comfortable enough to proceed. I then suggested that because there was no particular urgency, the patient could be referred to one of the four plastic surgeons in town the next day, which was no longer a holiday. Apparently, every fourth weekend this particular community did not have a plastic surgeon on call. Although this seemed to be odd to me, I suggested that I was sure that someone would be available the next day. It was then that I learned my second lesson.

The plastic surgeons did not accept referrals from the emergency department from the days when they were not on call! Therefore, the young man ended up driving for $4 \mathrm{~h}$ each way to have his finger dressed.

Unfortunately, this is not the only town near Toronto with a similar policy. What does this tell the general population and other physicians about plastic surgery? It reinforces the often heard perception that plastic surgeons do only beauty surgery. Is this really what all of us want to hear?
Mind you, I have nothing against aesthetic surgery and spent considerable time during my career teaching residents aesthetic surgery, but only as part of our specialty. I think that our graduates should become the best trained aesthetic surgeons.

I also have no problem with surgeons limiting their practice to aesthetic surgery in a private clinic. However, those surgeons who have hospital privileges must be available to provide their expertise to the community and their colleagues in other medical and surgical disciplines.

Another disturbing trend is the so called 'cherry-picking' practices we have noted over the past few years. There are a number of surgeons who no longer perform lower paying surgical procedures such as excisions of simple skin malignancies, minor hand procedures and, yes, abdomoinal lipectomies and, in some cases, reduction mammaplasties. Unfortunately, most of these patients end up at the doors of the teaching hospitals which, in reality, should be dealing with secondary or tertiary problems. Some of the excuses are rather interesting: "your residents need the practise!"

Now, what about the academic surgeons practising in these tertiary hospitals? We indeed have problems here as well. Some of the fault rests with the administrators, and some rests with us. Let me first look at the surgeons. There is no question that, over the past decade, our specialty has undergone significant metamorphosis, some of it good, some of it not so good!

The major change can be described as subspecialization. We are now maxillofacial surgeons, hand surgeons, cosmetic surgeons, burn surgeons, pediatric surgeons, breast reconstructive surgeons, head and neck reconstructive surgeons and microsurgeons, but no longer (with some exceptions) general plastic surgeons. I have no problem with some of the above, such as pediatric and burn surgery, because of the geographical and demographic situations. 
There is no question that this trend of subspecialization has improved both patient care and the profile of plastic surgery in general. However, as we learned during our recent University of Toronto plastic surgery retreat, in some institutions at least, we have not been given credit for these services and accomplishments!

The downside is the fact that our 'super-specialists' are either unwilling or unable to provide some of the basic supportive services for our large multidisciplinary hospitals.

Who is going to see the hospital inpatient with the pressure sore or the chronic wound, or the patient needing an urgent temporal artery biopsy? How about the suspected melanoma from our own family practice unit? We need to have and train a 'generalist' for each of our teaching hospitals.

I do recognize that we have a dilemma. We need the resources and the ability to hire new staff to rectify these problems. We need to get more involved in the hospital politics and make sure that we have the needed resources to provide at least the essential services!

At the same time, we must recognize the importance of a general plastic surgeon and treat general plastic surgery as if it were a subspecialty. Although as a general plastic surgeon I had a number of special interests (hobbies, if you wish) such as hand surgery, reduction mammaplasty and teaching aesthetic surgery, I thoroughly enjoyed the variety of my practice and would do it over again! However, when the time came to retire, it took us two years to find a replacement. Why? Because our residents were mostly exposed to the subspecialists who eventually became their role models and influenced the senior residents to follow their career paths. Of course, there is nothing wrong with that! However, we must be able to show our senior residents that we do need the generalists, and show them that it can be a very interesting, challenging and rewarding career, as was mine!

Now, so much for the criticisms. Here are some of my suggestions:

The community surgeons must realize that they have an important role to play in the community, not only to the patients but also to other care givers. They also have to realize that being on call one day in four or five is a privilege and not a hardship! Coming from someone who was on call one day in two for some $20+$ years, believe me it is true! They also have to realize that some of the 'mundane' procedures have to be performed even if they do not pay very much.

I do not think that it is appropriate to refer them to a tertiary care institution. Do your aesthetic surgery and enjoy your lifestyle but do not forget your roots and your specialty!

Academic surgeons and residency training program directors should recognize general plastic surgery as a subspecialty and encourage residents to pursue this career path. At the same time, they must take the time and effort to make sure that the administration is aware of the efforts and accomplishments of the plastic surgery division. They must be able to convince the hospital administrators and chiefs of surgery that in order to provide the essential services in a timely fashion, more resources are essential.

So, next morning, when you first look in the mirror, ask yourself "Have I done anything to help and preserve my specialty which has given me so much satisfaction both emotionally and economically?" 